\title{
FAST STOCHASTIC REORIENTATIONS IN NEMATIC PAA AND PAP
}

\author{
R. Podsiadly ${ }^{a}$, J. MAYeR ${ }^{b}$, J.A. JANiK ${ }^{b}$, J. KRAWCzYK ${ }^{b}$ AND T. STANeK ${ }^{a}$ \\ ${ }^{a}$ Faculty of Chemistry of the Jagiellonian University \\ Ingardena 3, 30-060 Kraków, Poland \\ ${ }^{b} \mathrm{H}$. Niewodniczański Institute of Nuclear Physics \\ Radzikowskiego 152, 31-342 Kraków, Poland
}

(Received October 22, 1996; revised version January 3, 1997)

Incoherent quasielastic neutron scattering spectra were measured for nematic para-azoxyanisole (PAA) and para-azoxyphenetole (PAP) with the energy resolution of $c a .30 \mu \mathrm{eV}$, at Dubna IBR-2 reactor. Data processing contained a careful background subtraction and the multiple scattering correction. The corrected spectra were subjected to a reorientational model fitting procedure. For PAA, a model of two convoluted reorientations that of benzene rings coupled with the terminals and that of the whole molecule around the long molecular axis - gave a fairly good description of the quasielastic neutron scattering spectrum. For PAP, introducing a third motion - interconformational jumps in the ethoxy terminals — in addition to motions suggested for PAA, gave a good agreement with the measured quasielastic neutron scattering spectrum.

PACS numbers: 24.50.Fq, 35.20. Yh, 61.30.Eb

\section{Introduction}

The incoherent quasielastic neutron scattering (QNS) method is widely used for determining the time scale and specification of fast stochastic reorientations of molecules and/or molecular fragments in condensed matter [1]. The interpretation of experimentally obtained QNS spectra takes place via fittings of a model scattering function convoluted with the instrumental function. The model scattering function contains characteristic times of those motions which were chosen in the reorientational model. The QNS method is selectively sensitive to hydrogen atoms, through which reorientations are observed. The method "works" in an appropriate "time window" eliminating the motions too slow and/or too fast. This occurs by an appropriate selection of the energy resolution of the neutron spectrometer.

If applied to complicated molecular systems the QNS method has to be used very carefully: not only the energy resolution but also the scattered neutron momentum transfer have to be properly selected, and all relevant corrections such as background subtraction and multiple scattering have to be properly estimated. A selective deuteration may also be helpful. All these precautions may, however, show up as-insufficient, if the system is too complicated.

This paper presents an application of the QNS method to molecular systems which are somewhat complicated, but still accessible to the method. The systems 


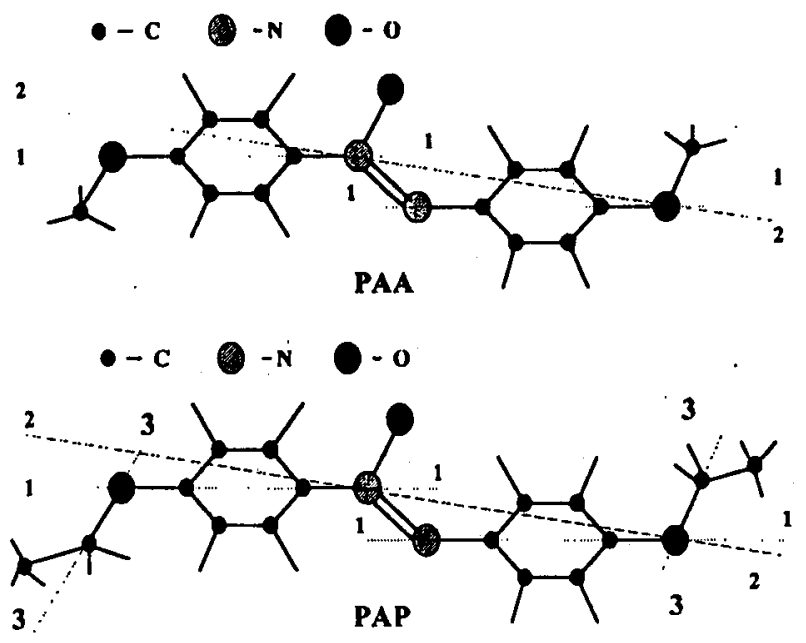

Fig. 1. The PAA and PAP molecules. 1..1, 2..2 and $3 . \ldots 3$ denote axes around which the reorientations as discussed in the model take place.

are: the nematic para-azoxyanisole (PAA) and the nematic para-azoxyphenetole (PAP), whose molecules are presented in Fig. 1. These substances were studied by us some time ago [2] with a broad energy resolution spectrometer. Now we present results obtained with a narrower energy resolution and with all experimental corrections more carefully taken into consideration.

\section{Experimental}

The QNS measurements with PAA and PAP were carried out on a time-of-flight "inverted geometry" spectrometer NERA, installed at the pulse reactor IBR-2 of the Joint Institute for Nuclear Research at Dubna, Russia [3]. The fixed scattered neutron energy was $4.76 \mathrm{meV}$. The energy resolution (HWHM of the instrumental function - explanation see below) was $c a .30 \mu \mathrm{eV}$. Measurements were carried out for several scattering angles, but the final analysis of data was performed only for one of them $-30^{\circ}$, which corresponded to the momentum transfer of $0.79 \AA^{-1}$. The choice of the momentum transfer was connected with the fact that the multiple scattering correction was smaller and that the higher order harmonics could have been neglected.

Unoriented samples were in an $\mathrm{Al}$-sample holder situated in a furnace with a thermostat. A sample thickness was $c a .1 .5 \mathrm{~mm}$. The multiple scattering was taken into consideration in the data analysis using the standard algorithm [4].

Isolation of QNS plus elastic components was performed by subtracting the most natural line connecting the far lying ends of the scattering pattern from the measured spectrum. 


\section{Scattering function and reorientational models} $[1,5-7]$

The theoretical function which was fitted to the isolated QNS spectra was

$$
F(\kappa, \omega)=\sigma^{\text {inc }} \frac{k_{f}}{k_{i}} \exp \left(\frac{\hbar \omega}{k_{\mathrm{B}} T}\right)[\widetilde{S}(\kappa, \omega) * G(\omega)],
$$

where $\sigma^{\text {inc }}$ is the incoherent cross-section of hydrogen ( 79.7 barns), $\hbar \kappa=\hbar k_{f}-\hbar k_{i}$ is the neutron momentum transfer (index $i$ stands for incoming neutron beam and $f$ for scattered), $\hbar \omega=E_{f}-E_{i}$ is the neutron energy transfer, $G(\omega)$ is the instrumental function, $*$ means the convolution operation, and $\widetilde{S}(\kappa, \omega)$ stands for the modified scattering function (explanation see below).

The instrumental function $G(\omega)$ is a spectrum measured for a certain sample which scatters the neutrons in a purely elastic way. (Due to a computational reason, the measured spectrum is usually transformed into an analytical form of a certain well-known mathematical function (into a Gaussian function, e.g.) by the procedure of the numerical fitting.)

In our experiment the spectra of the vanadium (measured at the room temperature for the convenient scattering angles) were taken as the instrumental functions. For the computer calculation they were transformed into a sum of the two Gaussian functions (due to a certain distortions of the spectra obtained from the NERA spectrometer - for detail explanation see [3]).

Formula (1) should contain the model scattering function $S(\kappa, \omega)$ corresponding to the type of reorientation under consideration. For instance, for a uniaxial rotational diffusion of hydrogen atoms the model scattering function $S(\kappa, \omega)$ is $[1,6,7]$ as follows:

$$
S(\kappa, \omega)=\frac{1}{3}\left\langle J_{0}^{2}(\kappa \cdot r)\right\rangle \delta(\omega)+\frac{2}{3 \pi} \sum_{n=1}^{\infty}\left\langle J_{n}^{2}(\kappa \cdot r)\right\rangle \frac{\Gamma_{n}}{\Gamma_{n}^{2}+(\hbar \omega)^{2}},
$$

where $J_{n}$ stands for cylindrical Bessel functions, $r$ is the gyration radius of the reorienting hydrogen atom, $\Gamma_{n}=\frac{\hbar n^{2}}{\tau_{1}}, \tau_{1}$ is the reorientational correlation time, and \langle\rangle stands for averaging over all molecular orientations in the sample.

For the uniaxial $120^{\circ}$ angular jumps the model scattering function $S(\kappa, \omega)$ is $[1,6,7]$

$$
S(\kappa, \omega)=\frac{1}{3}\left(1+\frac{2 \sin (\sqrt{3} \kappa \cdot r)}{\sqrt{3} \kappa \cdot r}\right) \delta(\omega)+\frac{2}{3 \pi}\left(1-\frac{\sin (\sqrt{3} \kappa \cdot r)}{\sqrt{3} \kappa \cdot r}\right) \frac{\Gamma}{\Gamma^{2}+(\hbar \omega)^{2}},
$$

where $\Gamma=\frac{3 \hbar}{2 \tau_{0}}$ and $\tau_{0}$ is the residence time between instantaneous jumps.

However, for molecules such as PAA or PAP one cannot expect that the scattering function will be so simple. Therefore we introduce in Eq. (1) the modified scattering function with a variable ratio of the elastic to quasielastic parts. This is achieved via a phenomenological parameter $p$

$$
\widetilde{S}(\kappa, \omega)=p \delta(\omega)+(1-p) S(\kappa, \omega) .
$$

The $p$-parameter can now be treated as a "detector" of the adequacy of the model. If $p=0$, it means that the model describes (formally) well the spectrum. If $p<0$, it means that some motions, whose time scale coincides with the time window 
of the spectrometer, have not been taken into account in the model. If $p>0$, it means that the model contains motions which in reality are not present in the sample.

\section{Results and discussion}

Figures 2a and $b$ present the isolated QNS spectra obtained for PAA and PAP (both nematic), respectively, at the scattering angle $30^{\circ}$. Spectra corrected for multiple scattering are shown as compared with the uncorrected ones. The fitting procedures were applied to the corrected spectra.
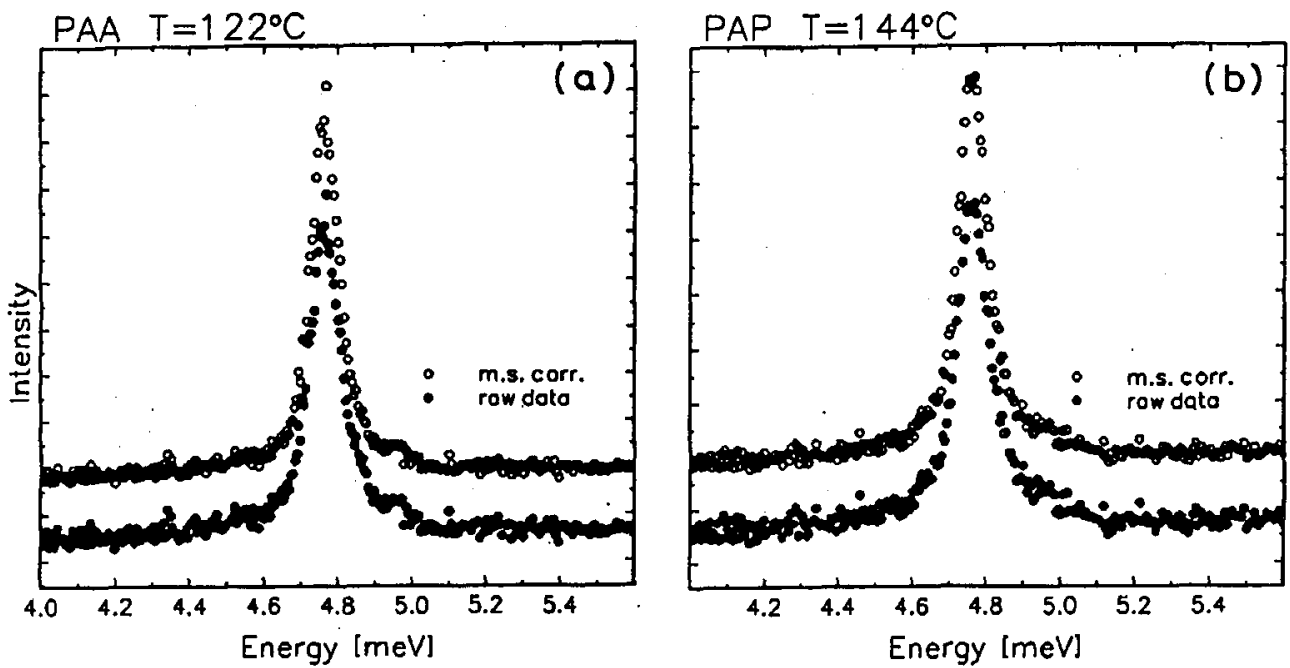

Fig. 2. QNS spectrum of PAA (a) and PAP (b) without and with the multiple scattering correction. The vertical separation of the spectra is for the sake of presentation only.

In the first attempt we tried the simplest model in which we assumed that a rotational diffusion of "molecular halves" is responsible for the QNS spectra. It means that the only motion taken into account is a rotation of the benzene rings (with the rigidly coupled terminal alkoxy groups) around the axes determined by the $\mathrm{C}$ (benzene)-N(azoxy) bonds (see Fig. 1). The results of this attempt are the following:

- for PAA (temp. $+120^{\circ} \mathrm{C}$ ): $\tau_{1}=8.5 \mathrm{ps}, \quad p=-0.19$,

- for PAP (temp. $+144^{\circ} \mathrm{C}$ ): $\tau_{1}=8.3 \mathrm{ps}, \quad p=-0.50$.

In both cases the $p$-parameter is significantly lower than zero, indicating the existence of more motions in the relevant time window.

Thus, we introduce to the model another motion in addition to that of the "halves". It is the rotational diffusion of the molecule as a whole, around the axis of the smallest moment of inertia (see Fig. 1). Now, we must point out that the 
correlation time for this motion is known from the dielectric relaxation measurements $[8,9]$ (here we assume that the reorientational correlation time is simply equal to the dielectric relaxation time).

The dielectric relaxation times for PAA and PAP (both nematic) are $22 \mathrm{ps}$ and $26 \mathrm{ps}$ respectively. Introducing these times as correlation times to the model, in which the scattering functions of the two motions (rotation of the "halves" and rotation of the whole molecule) are convoluted, we obtain

- for PAA (temp. $\left.+120^{\circ} \mathrm{C}\right): \tau_{1}$ (halves) $=7.7 \mathrm{ps}, \tau_{1}$ (whole) $=22 \mathrm{ps}[8]$, $p=0.09$,

- for PAP (temp. $+144^{\circ} \mathrm{C}$ ): $\tau_{1}$ (halves) $=3.9 \mathrm{ps}, \tau_{1}$ (whole) $=26 \mathrm{ps}[9]$, $p=-0.12$.

In the case of PAA we have to be satisfied. The value of $p$ is not very different from zero (which means that the model is adequate) and moreover, it is slightly positive, which would mean that we have introduced too many motions to the model. Perhaps the motions in question are in PAA somewhat restricted, due to steric conditions in the nematic phase, which caused an increase in the elastic component.

But in the case of PAP we still have one possibility of improvement. We may include into considerations the third motion, i.e. the angular $\left(120^{\circ}\right)$ jumps of the ethyl $\left(\mathrm{C}_{2} \mathrm{H}_{5}\right)$ segment of the ethoxy terminal around the axes determined by the $\mathrm{O}$ (ethoxy)-C(ethoxy) bonds (see Fig. 1). After performing time consuming convolution computation (with parametrized residence time of reorienting segments in the terminals [2]), we obtain
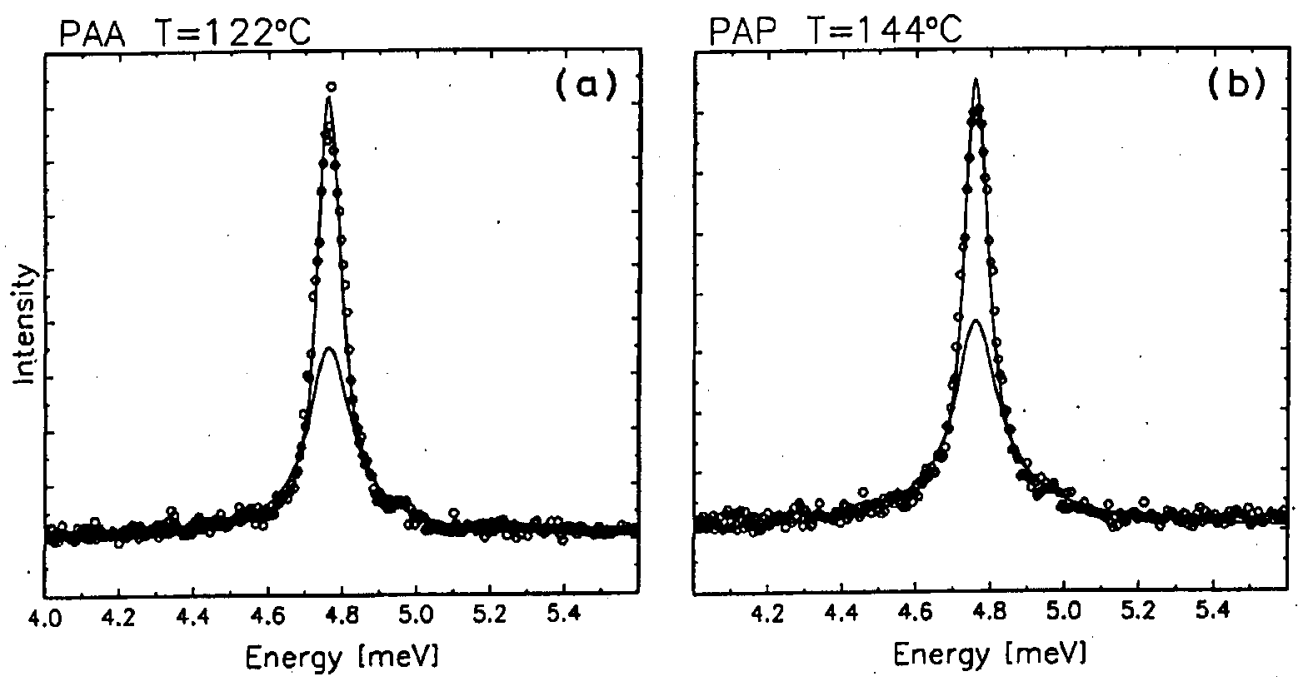

Fig. 3. Model fitting to the multiple scattering corrected QNS spectrum of PAA (a) and PAP (b). 
- for PAP (temp. $+144^{\circ} \mathrm{C}$ ): $\tau_{1}$ (halves) $=8.6 \mathrm{ps}, \quad \tau_{1}$ (whole) $=26 \mathrm{ps}[9]$, $\tau_{0}$ (in segment) $=10 \mathrm{ps}, p=0.01$.

It has to be pointed out here that the motion which obviously coincides with the time window of our spectrometer - the terminal methyl $\left(\mathrm{CH}_{3}\right)$ rotation around its triple symmetry axis - is not visible in our measurements at $30^{\circ}$ scattering angle, since the gyration radius of the hydrogen atoms is very small (ca. $0.9 \AA)$ and hence the intensity of the quasielastic component caused by it is negligible.

Figures $3 a$ and $b$ present the experimental spectra (corrected for multiple scattering) of PAA and PAP as compared with the model described as above.

\section{Conclusion}

The QNS spectra of the nematic PAA and PAP are fairly well described by the reorientational models, which contain: for PAA - a convolution of two motions - reorientation of molecular "halves" and reorientation of the whole molecule around its long axis, and, for PAP - a convolution of three motions two of them the same as for PAA and in addition the interconformational jumps in the ethoxy terminals.

\section{Acknowledgments}

Our thanks are due to Dr. I. Natkaniec from the I.M. Frank Laboratory of the Joint Institute for Nuclear Research at Dubna, for his valuable advises during the neutron measurements at the NERA spectrometer. We also thank Dr. W. Zając from the H. Niewodniczański Institute of Nuclear Physics in Kraków, for valuable discussions connected with the multiple scattering corrections.

This work was partially supported by the grant No. 2-P302-118-06 of the Committee for Scientific Research.

\section{References}

[1] T. Springer, Quasielastic Neutron Scattering for the Investigation of Diffusive Motions in Solids and Liquids, Springer Tracts in Modern Physics, Vol. 64, Springer Verlag, Berlin 1972.

[2] R. Podsiadły, J.A. Janik, Mol. Cryst. Liq. Cryst. 262, 285 (1995).

[3] I. Natkaniec, S.J. Briagin, J. Brańkowski, J. Mayer, in: Multicrystal Inverted Geometry Spectrometer NERA-PR at the IBR-2 Pulsed Reactor, Proc. ICANS XII, RAL Report 94-025, Vol. 1, Abington 1993, p. 89.

[4] M.W. Johnson, Harwell Report AERE-R 7682 (1974).

[5] P.S. Goyal, W. Nawrocik, S. Urban, J. Domosławski, I. Natkaniec, Acta Phys. Pol. A 46, 399 (1974).

[6] F. Volino, in: Microscopic Structure and Dynamics of Liquids, Eds. J. Dupuy, A.J. Dianoux, Plenum Publishing Corp., London 1978, p. 224.

[7] A.J. Leadbetter, R.M. Richardson, in: The Molecular Physics of Liquid Crystals, Eds. G.R. Luckhurst, G.W. Gray, Academic Press, London 1979, p. 171.

[8] S. Wróbel, J.A. Janik, J. Mościcki, S. Urban, Acta Phys. Pol. A 48, 215 (1975).

[9] S. Urban. S. Wróbel, K. Chłędowska, J. Chruściel, J.A. Janik, H. Kresse, Mol. Cryst. Liq. Cryst. 100, 57 (1983). 